\title{
Reproductive failure and reduced blood pressure in mice lacking the EP2 prostaglandin $E_{2}$ receptor
}

\author{
Stephen L. Tilley, ${ }^{1}$ Laurent P. Audoly, ${ }^{2}$ Elizabeth H. Hicks, ${ }^{1}$ Hyung-Suk Kim, ${ }^{3}$ \\ Patrick J. Flannery, ${ }^{2}$ Thomas M. Coffman, ${ }^{2}$ and Beverly H. Koller ${ }^{1}$ \\ ${ }^{1}$ Department of Medicine, University of North Carolina-Chapel Hill, Chapel Hill, North Carolina 27599-7248, USA \\ 2Department of Medicine, Duke University and Durham Veterans Affairs Medical Centers, Durham, North Carolina 27705, USA \\ ${ }^{3}$ Department of Pathology, University of North Carolina-Chapel Hill, Chapel Hill, North Carolina 27599-7525, USA
}

Address correspondence to: Beverly H. Koller, 7027 Thurston-Bowles Building, University of North Carolina-Chapel Hill, Chapel Hill, North Carolina 27599-7248, USA. Phone: (919) 962-2153; Fax: (919) 966-7524; E-mail: treawouns@aol.com.

Stephen L. Tilley and Laurent P. Audoly contributed equally to this work.

Received for publication February 17, 1999, and accepted in revised form April 13, 1999.

\begin{abstract}
Prostaglandins (PGs) are bioactive lipids that modulate a broad spectrum of biologic processes including reproduction and circulatory homeostasis. Although reproductive functions of mammals are influenced by PGs at numerous levels, including ovulation, fertilization, implantation, and decidualization, it is not clear which PGs are involved and whether a single mechanism affects all reproductive functions. Using mice deficient in 1 of 4 prostaglandin $\mathrm{E}_{2}\left(\mathrm{PGE}_{2}\right)$ receptors - specifically, the EP2 receptor - we show that $E p 2^{-/-}$females are infertile secondary to failure of the released ovum to become fertilized in vivo. Ep2 $2^{---}$ova could be fertilized in vitro, suggesting that in addition to previously defined roles, PGs may contribute to the microenvironment in which fertilization takes place. In addition to its effects on reproduction, $\mathrm{PGE}_{2}$ regulates regional blood flow in various vascular beds. However, its role in systemic blood pressure homeostasis is not clear. Mice deficient in the EP2 $\mathrm{PGE}_{2}$ receptor displayed resting systolic blood pressure that was significantly lower than in wild-type controls. Blood pressure increased in these animals when they were placed on a high-salt diet, suggesting that the EP2 receptor may be involved in sodium handling by the kidney. These studies demonstrate that $\mathrm{PGE}_{2}$, acting through the EP2 receptor, exerts potent regulatory effects on two major physiologic processes: blood pressure homeostasis and in vivo fertilization of the ovum.
\end{abstract}

J. Clin. Invest. 103:1539-1545 (1999).

\section{Introduction}

Prostaglandins (PGs) are well-recognized mediators of many important biologic processes (1). They exert their effects through binding to a family of $\mathrm{G}$ protein-coupled receptors (2). Among PGs, prostaglandin $\mathrm{E}_{2}\left(\mathrm{PGE}_{2}\right)$ is unique in that it acts through 4 different receptors (EP1-EP4), each with distinct but overlapping tissue distributions that activate different intracellular signaling pathways (3). It has been postulated that this complexity underlies the broad spectrum of physiologic responses that can be mediated by $\mathrm{PGE}_{2}$ and that specific receptors will be associated with each of these responses (4).

A role for PGs in reproductive physiology has been recognized for many years. PGs are present throughout the female reproductive tract, and the entire reproductive process is believed to be under the influence of these lipid mediators (5). Early studies showed that several aspects of female reproduction could be affected by inhibitors of PG synthesis $(6,7)$. Furthermore, genes encoding prostanoid receptors are expressed in both a temporal and cell-specific fashion during key events of early pregnancy $(8,9)$. More recently, mice deficient in cyclooxygenase-2 (COX2), the rate-limiting enzyme in synthesis of all prostanoids during pregnancy, including $\mathrm{PGE}_{2}$, have been generated. These mice are infertile, with abnormalities in ovulation, fertilization, implantation, and decidualization (10). However, because COX-2 is critical for the production of $\mathrm{PGE}_{2}$,
$\mathrm{PGD}_{2}, \mathrm{PGF}_{2}, \mathrm{PGI}_{2}$ (prostacyclin), and thromboxane $\mathrm{A}_{2}$, these studies did not identify the particular prostanoid or receptor critical to each of the reproductive functions disrupted in these animals. Some insight into the mechanism by which prostanoids mediate their effects is beginning to emerge from the study of animals deficient in specific receptors. For example, mice deficient in the receptor for PGF (FP receptor) demonstrated failure of parturition but showed no abnormalities in ovulation, fertilization, or implantation (11).

Surprisingly, no decreased fertility has been reported for the EP3-, EP4-, thromboxane (TP)-, or prostacyclin (IP)receptor-deficient mice, despite the expression of these prostanoid receptors in the reproductive tract $(8,9,12-16)$.

$\mathrm{PGE}_{2}$ also has potent effects on the cardiovascular system. A role for $\mathrm{PGE}_{2}$ in blood pressure homeostasis has been recognized for years, but its effects are complex because it acts on multiple tissues important to the maintenance and control of blood pressure (17). Although it is clear that blood pressure homeostasis and many aspects of female reproduction may be regulated by PGs, the precise contribution of the individual receptors that mediate the actions of $\mathrm{PGE}_{2}$ remains to be defined. Utilizing mice deficient in the EP2 receptor, we show that this PG receptor plays a critical role in successful fertilization of the released ovum and the maintenance of circulatory homeostasis. 


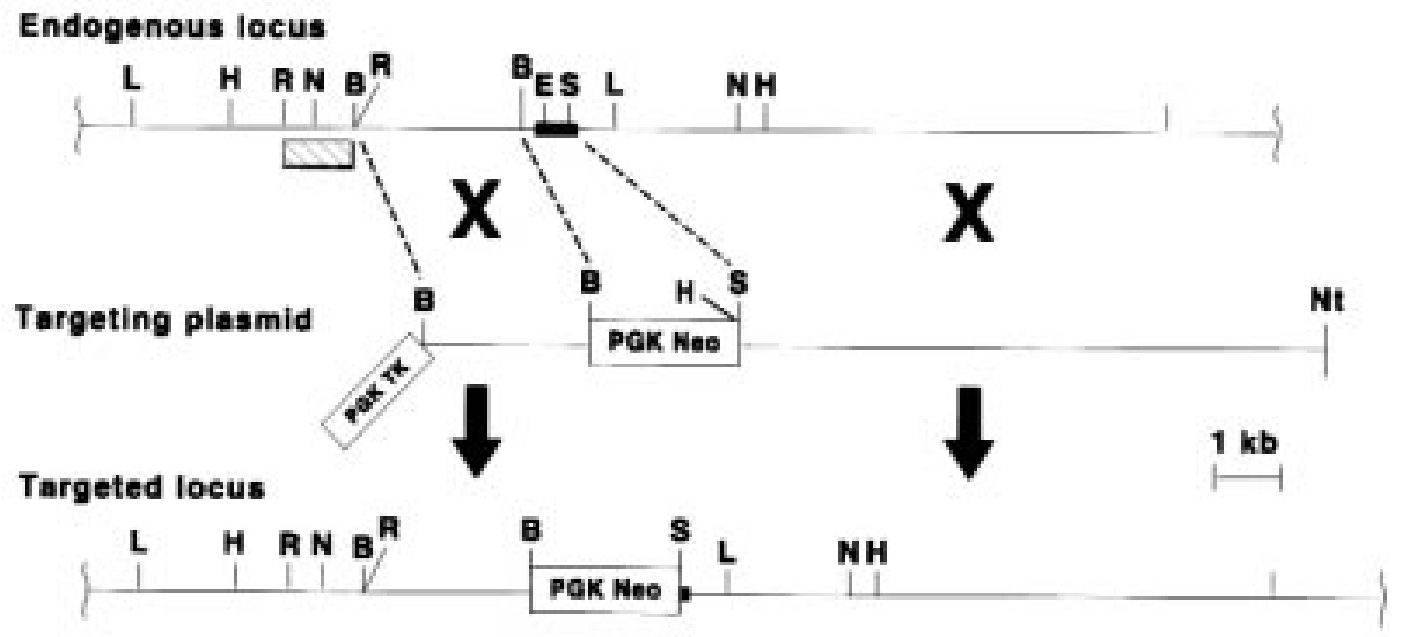

b

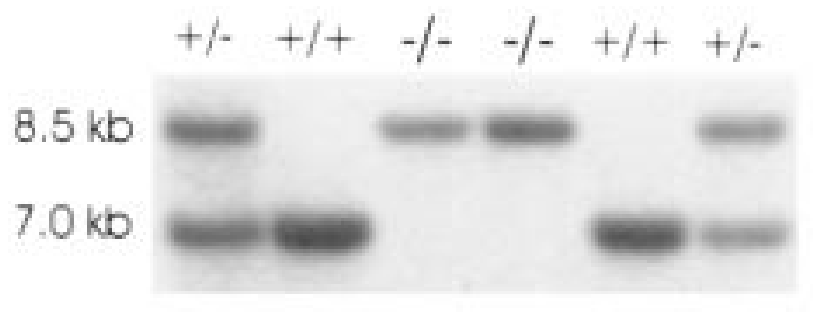

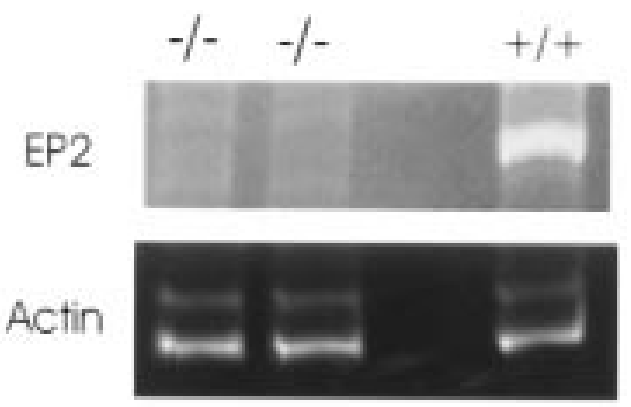

\section{Figure 1}

Disruption of the gene encoding the EP2 receptor. (a) Restriction maps of the targeting construct, endogenous locus, and targeted locus. The dark filled box represents exon sequences of the Ep2 gene. The open boxes represent phosphoglycerate kinase-thymidine kinase (PGK-TK) and PGK-Neo selection cassettes. Homologous recombination of the targeting vector with the endogenous locus results in deletion of a 355-bp segment of DNA that is predicted to encode 3 transmembrane domains and 2 intracellular loops of mature EP2 protein. The hatch filled box represents the probe used to detect homologous recombination events by Southern blot analysis. Relevant restriction sites are abbreviated by the following: B, Bam HI; E, Eagl; H, HindIII; L, Bgll; N, Nsil; Nt, Notl; R, EcoRI; S, Sacll. Note that not all Hindlll sites are mapped. (b) Southern blot analysis of DNA obtained from tail biopsies of offspring from $E p 2^{+/-}$heterozygote breedings. HindIII-digested DNA was analyzed using a 1.1-kb EcoRI probe corresponding to DNA outside the targeted region of the Ep2 gene. This probe detects a 7-kb band derived from the endogenous locus and an 8.5-kb band from the targeted locus. (c) RT-PCR ampli-

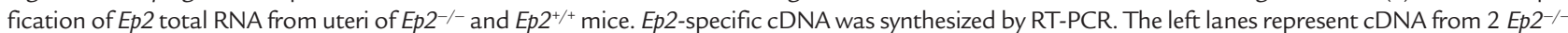
animals; the lane on the far right represents cDNA from an $E p 2^{+/+}$animal. To ensure that the failure to detect an $E p 2$-specific DNA fragment in the mRNA obtained from Ep2-/- animals was not due to the absence of RNA, the same samples were subjected to RT-PCR using actin-specific primers.

\section{Methods}

Animal welfare. The use of experimental animals was in accordance with the Institutional Animal Care and Use Committee (IACUC) guidelines of the University of North Carolina-Chapel Hill and Duke University.

Generation of Ep2 $2^{-/-}$mice. Genomic clones were isolated from a 129/Sv mouse genomic library with an Ep2 cDNA probe and their identity confirmed by sequence analysis. A targeting vector was constructed in which the DNA encoding amino acids 246-601 was replaced by a neomycin-resistant gene and electroporated into 129/Ola-derived E14TG2a embryonic stem (ES) cells, and neomycin- and ganciclovir-resistant colonies were identified using standard methods (18). DNA isolated from ES cell colonies was digested with the restriction enzyme HindIII and analyzed by Southern blot to identify clones with a targeted Ep2 allele. Chimeras derived from targeted ES cells were mated with $129 / \mathrm{SvEv}$ mice, and offspring carrying the targeted allele were identified by Southern blot analysis. These heterozygotes were intercrossed to produce mice homozygous for the Ep2 mutation.

Analysis of Ep2 RNA expression. Total uterine RNA was isolated from 7-week-old $\mathrm{Ep}^{2^{-/}}$and $E \mathrm{P}^{+/+}$mice using RNAzol (TelTest Inc., Friendswood, Texas, USA) according to the manufacturer's specifications. Ep2 and actin cDNA were synthesized by reverse transcription, and PCR amplification was carried out using the following oligonucleotide primers: Ep2-1F (5'-GTGGCCCTGGCTCCCGAAAGTC- $\left.3^{\prime}\right), \quad$ Ep2-2R (5'-GGCAAG-GAGCATATGGCGAAGGTG-3'), actin-1F( $5^{\prime}$-TAAGGCCAACCGTGAAAAGAT GAC-3'), and actin-2R (5'-ACCGCTCGTTGCCAATAGTGATG-3'). The PCR products were analyzed by gel electrophoresis and staining with ethidium bromide.

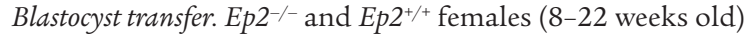
were mated with wild-type vasectomized males and checked daily for vaginal plugs. Fourteen to 20 C57BL/ 6 blastocysts were transferred into the uteri of recipient females on day 3.5 


\section{Figure 2}

Ovulation and fertilization in Ep2 $2^{-/-}$and $E p 2^{+/+}$mice.

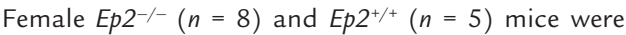
mated with wild-type males, and ovulation and in vivo fertilization were determined by counting eggs and embryos 2 days following coitus. For in vitro fertilization, eggs from mice of both genotypes were collected 12 hours after ovulation and cultured with sperm obtained from wild-type males. Fertilization was scored by counting the number of 2-cell embryos 24 hours later. ${ }^{*} P<0.0001$.

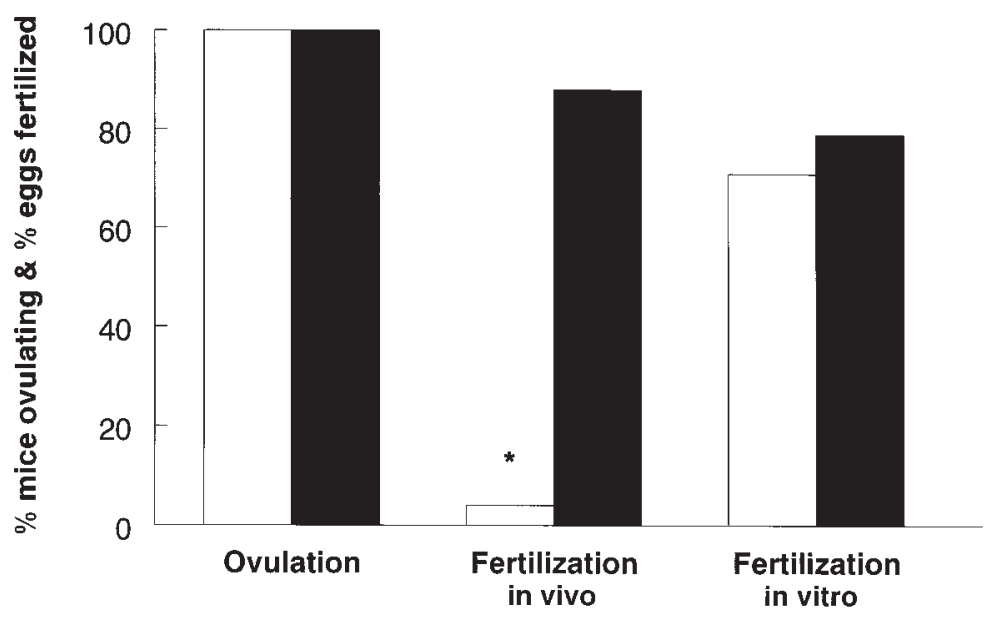

Statistical analysis. Data are presented as mean \pm SEM. Statistical significance was assessed by using $\chi^{2}$ analysis and the unpaired Student's $t$ test. daily for pregnancy and delivery.

Ovulation and fertilization. Ep2 $2^{-/-}$and $\mathrm{Ep}^{2^{+/+}}$mice were mated with wild-type males, and inspection for vaginal plugs was performed daily. On the day after finding a vaginal plug, mice were sacrificed and oviducts were flushed with $100-200 \mu \mathrm{L}$ of $\mathrm{CO}_{2-}$ independent media to recover eggs or embryos.

In vitro fertilization. Female mice were superovulated by injecting 5 IU pregnant mare's serum intraperitoneally and then, 48 hours later, injecting 5 IU human chorionic gonadotropin (hCG) intraperitoneally. Twelve hours after hCG was injected, mice were sacrificed and the ova were collected and incubated in HTF media for $4-6$ hours at $37^{\circ} \mathrm{C}$ with sperm collected from the epididymides of proven male mice. The ova were then washed and cultured for an additional 24 hours, at which time the number of unfertilized eggs and 2-cell embryos was determined.

Blood pressure measurements. Resting systolic blood was measured in conscious female mice (3-4 months old) using a computerized noninvasive tail-cuff system (Visitech Systems, Apex, North Carolina, USA). The validity of this system has been demonstrated previously (19). Mice were adapted to the system for 5 days, after which blood pressure was recorded daily for 5 consecutive days after 2 weeks of either a normal $(0.4 \% \mathrm{NaCl})$ or high-salt diet (6\% NaCl; Harlan Teklad Laboratory, Madison, Wisconsin, USA), ad libitum, with free access to water.

Plasma electrolytes. Blood was collected by cardiac puncture into cold EDTA-coated tubes, and plasma samples were immediately placed at $-80^{\circ} \mathrm{C}$. Plasma sodium levels were determined by flame photometry.

Twenty-four-hour urine collections for $P G E_{2}$ determination. Urine was collected over a 24-hour period using a mouse metabolic cage, and stored at $-20^{\circ} \mathrm{C}$ immediately upon completion of the study period. Urinary $\mathrm{PGE}_{2}$ was determined by enzyme immunoassay according to the manufacturer's protocol (Cayman Chemicals, Ann Arbor, Michigan, USA).

Plasma renin activity. Plasma renin concentration was determined by radioimmunoassay according to the manufacturer's protocol (Du Pont NEN Research Products, Boston, Massachusetts, USA).

Renin mRNA. Kidneys were harvested from $E p 2^{-/-}$and $E p 2^{+/+}$ mice, frozen in liquid nitrogen, and immediately placed at $-80^{\circ} \mathrm{C}$. Total RNA was extracted by homogenization with RNAzol (Tel-Test Inc.) according to the manufacturer's specifications. Renin mRNA was measured by RNase protection assay as described previously (20).

\section{Results}

Generation of Ep2 $2^{-1-}$ mice. Mouse ES cells in which the Ep2 gene was disrupted by homologous recombination were generated using the scheme shown in Figure 1a. Chimeric animals created with these targeted cells were mated with $129 / \mathrm{SvEv}$ mice to generate heterozygotes for the mutant allele on the 129 background. Heterozygous $E p 2^{+/-}$mice were intercrossed and $E p 2^{-/-}$offspring were born at expected frequencies $-25.2 \%\left(E p 2^{+/+}\right), 50 \%\left(E p 2^{+/-}\right)$, and $24.7 \%\left(E p 2^{-/-}\right)$- indicating that this receptor does not have a unique role in intrauterine development or perinatal survival (Figure 1b). The appearance, behavior, and longevity of $\mathrm{Ep}^{-{ }^{--}}$mice did not differ from that of wildtype littermates, with some mice living longer than 18 months. Gross and histologic appearance of all major organs, including the kidney, uterus, and ovaries, did not differ between $\mathrm{Ep}^{-\gamma^{--}}$and wild-type animals. Loss of Ep2 expression was verified by RT-PCR analysis of RNA from $E p 2^{-/-}$animals (Figure 1c).

Fertility of the EP2-deficient animals. Whereas Ep2 $2^{-/-}$males

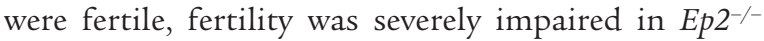
females. After 12 weeks of mating, only 6 of 34 (18\%) $E 2^{-1-}$ females achieved pregnancy and delivered litters, compared with 39 of $42(93 \%) E p 2^{+/-}$females $(P=0.001)$. This reduced fertility of the Ep $2^{---}$females did not reflect a decrease in the frequency of mating, as determined by the presence of vaginal plugs.

Blastocyst transfer. To determine if reproductive failure was due to defective implantation of the embryos, wildtype blastocysts were transferred into the uteri of $\mathrm{Ep}^{-2^{--}}$ and $E p 2^{+/+}$animals on day 3.5 of pseudopregnancy. As shown in Table 1, eight successful pregnancies were achieved through blastocyst transfer into Ep2 $2^{-/-}$females, with 60 out of 191 blastocysts developing into embryos (31\% efficiency). These results are not significantly different from those obtained in $E p 2^{+/+}$females (Table 1). Gestational time was similar for both groups of animals 
Figure 3

PRA in $E p 2^{-/-}$and $E p 2^{+/+}$mice on normal and high-salt diets. Plasma was prepared from Ep2-/- $(n=8)$ and $E p 2^{+/+}(n=7)$ mice on a normal diet $(0.4 \% \mathrm{NaCl})$, and PRA was determined by radioimmunoassay. PRA was also measured in $\mathrm{Ep}^{-/-}(n=5)$ and $E p 2^{+/+}(n=6)$ mice after 10 days on a high-salt diet $(6 \% \mathrm{NaCl})$. Data represent mean \pm SEM

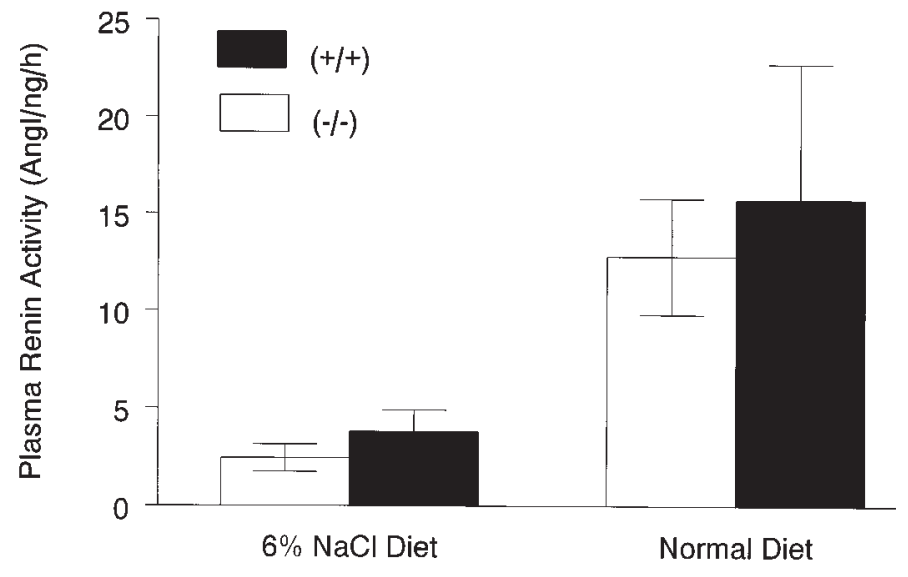

ulated release of eggs to a similar extent in the 2 groups of animals $(11.8 \pm 1.9$ vs. $15.6 \pm 1.9 ; P=0.165)$

Fertilization, however, was severely impaired in $E \mathrm{p}^{-1-}$ mice. Only 4\% (2/55) of eggs from $8 E \mathrm{Ep}^{-/-}$mice were fertilized, compared with $88 \%(38 / 43)$ of eggs from $5 \mathrm{Ep}^{+/+}$ mice $(P<0.0001)$ (Figure 2). Morphologically, eggs and their cumulus complexes from $E p 2^{-/-}$animals were indistinguishable from those of wild-type controls. Motile sperm were present in the oviducts of $E \mathrm{p}^{-/-}$females and wild-type females 12 hours postcoitus, suggesting that alterations in sperm transport were not the underlying

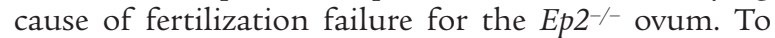
exclude the possibility that fertilization failure was secondary to delayed ovulation, experiments were repeated with mice in which ovulation was hormonally induced. Whereas 64 of 102 eggs released from $6 \mathrm{Ep}^{+/+}$females were fertilized, none of the 61 eggs released from $5 \mathrm{Ep} 2^{-/-}$ females were fertilized $(P<0.001)$.

To determine whether fertilization failure was secondary to abnormalities of the ovum, in vitro fertilization was performed with ova from $E p 2^{-/-}$and $E p 2^{+/+}$mice. Seventy-one percent of eggs obtained from $4 \mathrm{Ep}^{2^{-/-}}$females were successfully fertilized in vitro, similar to in vitro fertilization rates in control eggs (Figure 2).

Blood pressure regulation. To define the role of the EP2 receptor in regulating blood pressure, we compared systolic blood pressure in conscious $\mathrm{Ep}^{-2^{--}}$and $\mathrm{Ep}^{+/+}$ mice. In animals maintained on a normal diet $(0.4 \%$ $\mathrm{NaCl}$ ), systolic blood pressure was reduced significantly in the $E p 2^{-/-}$mice compared with wild-type controls $(109 \pm 3$ vs. $122 \pm 5 \mathrm{mmHg} ; P=0.04)$ (Table 2$)$. To determine whether this blood pressure difference could be overcome by providing excess sodium in the diet, we fed $E p 2^{-/-}$and $E p 2^{+/+}$mice a high-salt diet containing 6\% $\mathrm{NaCl}$. This alteration of dietary $\mathrm{NaCl}$ content had no effect on blood pressure in the wild-type mice. In contrast, blood pressure increased significantly in $\mathrm{Ep}^{-/-}$ mice on a high-salt diet, from $109 \pm 3$ to $117 \pm 3 \mathrm{mmHg}$ $(P=0.01)$. Plasma sodium levels and urinary $\mathrm{PGE}_{2}$ excretion were no different between $E \mathrm{p}^{2^{-/-}}$and $E \mathrm{p}^{2^{+/+}}$ animals (data not shown).

To evaluate the effect of the Ep2 mutation on regulation of the renin-angiotensin system, and its potential contribution to altered blood pressure regulation, we also measured plasma renin activity (PRA) in the 2 
groups of mice on the different dietary $\mathrm{NaCl}$ regimens. Figure 3 shows the changes in PRA measured in mice on normal and high-salt diets. Despite the differences in blood pressure between $E p 2^{-/-}$and $\mathrm{Ep}^{+/+}$mice on a normal $(0.4 \% \mathrm{NaCl})$ diet, PRAs were virtually identical $(12.4 \pm 3.0[n=8]$ vs. $15.8 \pm 7.0[n=7] \mathrm{Ang} \mathrm{I} / \mathrm{ng} / \mathrm{h}$; $P=0.63$ ) (Figure 3). Renin mRNA levels were also similar between genotypes $(26.9 \pm 5.6 \mathrm{vs} .25 .5 \pm 1.5 \mathrm{pg} / \mu \mathrm{g}$ total RNA) (Figure 4). After 10 days on the high-salt diet, PRA was suppressed appropriately in both Ep2 $2^{-/-}$ and $E p 2^{+/+}$mice $(2.4 \pm 0.7[n=5]$ vs. $3.8 \pm 1.1[n=6]$ Ang $\mathrm{I} / \mathrm{ng} / \mathrm{h} ; P=0.34$ ) (Figure 3 ).

To determine if the reproductive failure of $\mathrm{Ep}^{-/-}$ females was related to the reduced blood pressure of these animals, fertilization was examined after 10 days on a high-salt diet $(6 \% \mathrm{NaCl})$. Only $3 \%$ of eggs from $\mathrm{Ep}^{-1-}$ mice were fertilized, compared with $93 \%$ of eggs from $E p 2^{+/+}$mice $(n=6 ; P<0.0001)$. These results suggest that the impaired fertilization in $E p 2^{-/-}$females is independent of the hemodynamic changes seen in these animals.

\section{Discussion}

We report here on the generation of mice deficient in the expression of the $\mathrm{PGE}_{2} \mathrm{EP} 2$ receptor. Although these mice are generally healthy, female Ep $2^{-/-}$mice have significantly reduced pregnancy rates, and when these animals do become pregnant, they deliver smaller litters than $E 2^{+/-}$and wild-type controls. We show that this decrease in fertility is primarily due to a significantly lower fertilization rate in the Ep2 $2^{-/-}$females. This reduction in fertilization is not due to an intrinsic defect of the ovum, because the in vitro fertilization rates of the $E \mathrm{p}^{-{ }^{--}}$and wild-type ova are identical. In addition to this decreased fertility, we show that Ep $2^{-/-}$animals have reduced blood pressure on normal diets and that this hypotension can be partially ameliorated by increased dietary salt.

Although all 4 EP receptors are expressed in the uterus, reduced fertility has not been reported for either the EP3- or EP4-deficient mice $(13,16)$. Although the fertility of the EP2-deficient mouse is decreased, implantation

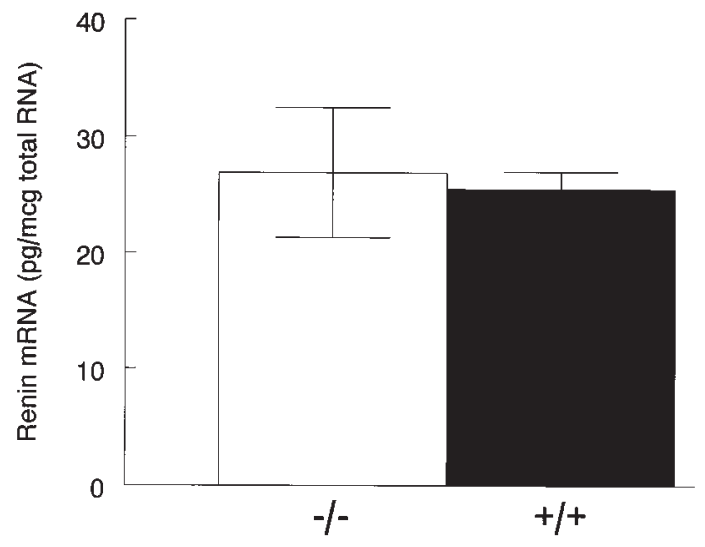

Figure 4

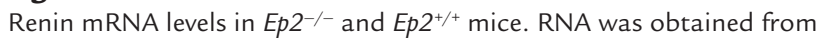

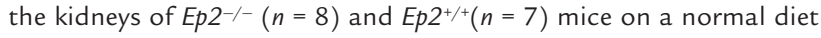
$(0.4 \% \mathrm{NaCl})$, and renin mRNA levels were determined by RNase protection assay. Data represent mean \pm SEM.
Table 2

Average resting systolic blood pressure of Ep2 $2^{-/-}$and $E p 2^{+/+}$mice

\begin{tabular}{ccccc}
\cline { 2 - 3 } Diet & $\mathrm{Ep2}^{-/-}$ & $n$ & $\mathrm{Ep} 2^{+/+}$ & $n$ \\
$\mathrm{ND}^{\mathrm{A}}$ & $109 \pm 3^{\mathrm{C}, \mathrm{D}}$ & 14 & $122 \pm 5^{\mathrm{C}}$ & 13 \\
$\mathrm{HD}^{\mathrm{B}}$ & $117 \pm 3^{\mathrm{D}}$ & 14 & $126 \pm 4$ & 14
\end{tabular}

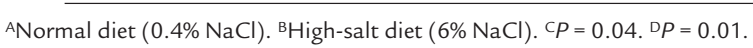

and decidualization is identical to that seen in wild-type animals. Decreased implantation or decidualization was not reported for the FP-, IP-, or TP-receptor-deficient mice $(11,12,21)$. These observations are somewhat surprising given the abnormalities of these reproductive functions in the COX-2-deficient mice. A number of explanations for these findings can be put forth. First, it is possible that $\mathrm{PGD}_{2}$ or $\mathrm{PGE}_{2}$ activation of $\mathrm{EP} 1$ is important for implantation. It is also possible that, despite the seemingly unique pattern of expression of the various prostanoid receptors, their functions overlap enough that implantation is not affected by the loss of a single receptor. Alternatively, a yet to be identified COX-2 product might be important for implantation.

Although ovulation is primarily triggered by a surge of luteinizing hormone, a role for PGs in this process has also been suggested. For example, inhibitors of PG synthesis, such as indomethacin, can prevent follicular rupture, and this effect can be overcome by administration of $\mathrm{PGE}_{2}$ (22). Similarly, mice deficient in COX-2 demonstrated impaired ovulation (10). Our results indicate that these effects of PGs on ovulation are not mediated through the EP2 receptor, because both natural and hormonally induced ovulation in $E \mathrm{p}^{-/-}$females were no different from wild-type controls.

Although PGs are produced throughout the female reproductive tract and by ovulated cumulus cell-oocyte complexes, their precise role in the fertilization process remains unclear (23). Again, direct evidence for a role of prostanoids in fertilization came from studies of the COX-2-deficient mice. The results presented here are consistent with the hypothesis that at least one function of prostanoids in fertilization is mediated by $\mathrm{PGE}_{2}$ through the EP2 receptor. To define the mechanism by which loss of the EP2 receptor leads to decreased fertilization, a number of experiments were carried out. To determine if decreased fertilization was due to delayed or premature release of the ovum, ovulation was induced by administration of hCG, and the appearance of the eggs in the oviduct, as well as the rate of fertilization, was examined. Although the appearance of the ampulla in the oviduct was similar, the ova released after the animals were treated with hCG still failed to become fertilized. In addition, ova and their cumulus complexes from Ep2 $2^{-/-}$ animals were morphologically indistinguishable from those from wild-type controls. Therefore, it seems unlikely that the decreased fertilization of eggs from $E \mathrm{p}^{-/-}$mice results from an alteration in the timing of ovulation.

The fertilization process requires successful transport of sperm to the oviducts, where they can be sequestered for interaction with the ova. PGs have been shown to facilitate sperm transport through the female reproductive tract, not only by their actions on sperm but also by their effects 
on female reproductive tissue, including oviductal smooth muscle (24). Numerous sperm were found in the oviducts of Ep2 $2^{-/-}$females within 12 hours of coitus, making it unlikely that alteration in sperm transport is the cause of fertilization failure in the EP2-deficient animals.

Together, these findings suggest that the reduced fertilization seen in these animals could be a function of PGs intrinsic to the ovum and cumulus cells, or that EP2mediated $\mathrm{PGE}_{2}$ functions are essential for establishing an oviductal microenvironment conducive to successful fertilization. To distinguish between these 2 possibilities, we compared in vitro and in vivo fertilization of the Ep $2^{-/-}$ ovum. Successful in vitro fertilization of ova from $E \mathrm{p}^{-/-}$ animals does not support the former hypothesis. These results are consistent with studies showing that maturation of the ovum is independent of PGs $(25,26)$. Previous studies have also suggested that ovum viability is unaffected by inhibitors of PG synthesis in vitro (5).

Our studies are consistent with the hypothesis that $\mathrm{PGE}_{2}$ acts through the $\mathrm{EP} 2$ receptor to define a microenvironment in the oviduct conducive to fertilization. Although such a function in reproductive physiology has not previously been ascribed to these lipids, it is consistent with the diverse actions of PGs in numerous organ systems. For example, PGs have been shown to modify ion transport in a number of epithelia $(27,28)$. It is possible that the ionic composition of the oviductal fluid is altered in the E $\mathrm{p}^{-/-}$animals and that this, in turn, leads to reduced fertilization.

$\mathrm{PGE}_{2}$ also has potent effects on the cardiovascular system. A role for $\mathrm{PGE}_{2}$ in blood pressure homeostasis has been recognized for years, but these actions are complex, involving regulation of vascular tone and sodium balance (17). To examine the role of the EP2 receptor in blood pressure regulation, we measured systolic blood pressure in conscious $E \mathrm{p}^{-/-}$animals. On a normal diet $(0.4 \% \mathrm{NaCl})$, systolic blood pressure was reduced by 13 $\mathrm{mmHg}$ in the EP2-deficient mice compared with controls. To determine whether this difference in blood pressure could be overcome by providing excess sodium in the diet, $E p 2^{-/-}$and $E p 2^{+/+}$mice were fed high-salt diets containing $6 \% \mathrm{NaCl}$ (wt/wt). This increase in dietary $\mathrm{NaCl}$ content had no effect on blood pressures in the wild-type mice. In contrast, on the high-salt diet, blood

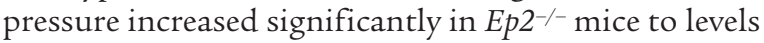
that were not significantly different than controls. Thus, reduced blood pressure in the Ep2 $2^{-/-}$mice is sodium sensitive and can be overcome with dietary sodium loading. This suggests that the absence of EP2 receptors produces an abnormality in sodium handling by the kidney. EP2 receptor expression in the kidney has been demonstrated by both RT-PCR and Northern analysis $(29,30)$, although its cellular functions in the kidney have not been clearly elucidated.

Our findings of reduced blood pressure in the Ep2-/animals may seem somewhat paradoxical given the known vasorelaxant actions of $\mathrm{PGE}_{2}(31,32)$. However, whereas acute infusions of $\mathrm{PGE}_{2}$ cause vasodilation, chronic infusions of $\mathrm{PGE}_{2}$ increase blood pressure through direct stimulation of renin secretion (33). Furthermore, inhibitors of PG synthesis have been shown to lower blood pressure and renin levels in patients with renovascular hypertension (34). It is well established that PGs can stimulate renin release through actions on the juxtaglomerular apparatus; hormones that increase intracellular cAMP levels stimulate renin release by these specialized cells (35). Because 2 EP receptors, EP2 and EP4, are $\mathrm{G}_{\mathrm{s}}$ protein-coupled, it has been suggested that one or both of these receptors may mediate this action of $\mathrm{PGE}_{2}(28,36)$.

To determine whether abnormal regulation of the renin-angiotensin system might contribute to altered

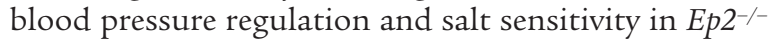
mice, we measured PRA in these animals. Despite their lower blood pressures while on a normal diet, neither

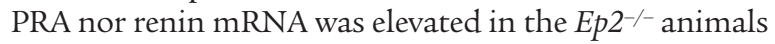
(Figures 3 and 4). This differs from other lines of mice harboring targeted mutations that lower blood pressure, where PRA and renin mRNA are consistently elevated as a compensatory mechanism $(20,37)$. These findings suggest that renin responses are significantly impaired in EP2-deficient mice. Failure to stimulate PRA may contribute to the reduced blood pressure seen in these animals while they are on a normal diet.

The contribution of PGs to many important biologic processes has been recognized for years. Recently, mice carrying homozygous null mutations for genes encoding enzymes in the PG biosynthetic pathway and in PG receptors themselves have furthered our understanding of the role that these lipid mediators play in various aspects of reproduction $(10,11)$. The role of PGs in maintaining circulatory homeostasis has been more controversial and less understood. Here we define critical roles for the EP2 $\mathrm{PGE}_{2}$ receptor in blood pressure regulation and female reproduction. Specifically, we present evidence supporting the hypothesis that $\mathrm{PGE}_{2}$, acting though the EP2 receptor, is important for establishment of the microenvironment required for successful fertilization of the released ovum. We also show that the EP2 receptor is essential for normal blood pressure homeostasis. Mice lacking this receptor are relatively hypotensive, and this hypotension can be partially reversed by salt loading. Finally, compensatory increases in renin are not seen in these animals, suggesting that the EP2 receptor may be involved in PG-stimulated renin release. Continued investigations into the specific roles of the receptors that mediate the actions of PGs will further our understanding of these important lipid mediators and potentially may lead to the development of more specific and less toxic therapies for a variety of disease processes.

Note added in proof. During the review process of this manuscript, an analysis of EP2 receptor-deficient mice was independently reported in the February 1999 issue of Nature Medicine (5:217-220).

\section{Acknowledgments}

The authors thank Cun-Yu Wang for his work on the targeting construct; Anne Latour for her work in tissue culture; Betsy Drake and Jane Farley (The Jackson Laboratory, Bar Harbor, Maine, USA) for their advice on in vitro fertilization; and M. Solle, J.E. Fabre, and J. Snouwaert for their helpful comments on the manuscript. This work was supported in part by National Institutes of Health grant HL-58554. 
1. Oates, J.A., et al. 1988. Clinical implications of prostaglandin and thromboxane $\mathrm{A}_{2}$ formation. N. Engl. J. Med. 319:689-698.

2. Coleman, R.A., Smith, W.L., and Narumiya, S. 1994. International union of pharmacology classification of prostanoid receptors: properties, distribution, and structure of the receptors and their subtypes. Pharmacol. Rev. 46:205-229.

3. Negishi, M., Sugimoto, Y., and Ichikawa, A. 1995. Molecular mechanisms of diverse actions of prostanoid receptors. Biochim. Biophys. Acta. 1259:109-120.

4. Ashby, B. 1994. Interactions among prostaglandin receptors. Receptor. 4:31-42.

5. Hayashi, S., Noda, Y., and Mori, T. 1988. Analysis of the role of prostaglandins in the fertilization process. Eur. J. Obstet. Gynecol. Reprod. Biol. 29:287-297.

6. Kennedy, T.G. 1977. Evidence for a role for prostaglandins in the initiation of blastocyst implantation in the rat. Biol. Reprod. 16:286-291.

7. Tsafriri, A., Lindner, H.R., Zor, U., and Lamprecht, S.A. 1972. Physiologic role of prostaglandins in the induction of ovulation. Prostaglandins. 2:1-10.

8. Yang, Z.-M., et al. 1997. Potential sites of prostaglandin actions in the periimplantation mouse uterus: differential expression and regulation of prostaglandin receptor genes. Biol. Reprod. 56:368-379.

9. Katsuyama, M., et al. 1997. Distinct cellular localization of the messenger ribonucleic acid for prostaglandin E receptor subtypes in the mouse uterus during pseudopregnancy. Endocrinology. 138:344-350.

10. Lim, H., et al. 1997. Multiple female reproductive failures in cyclooxygenase 2-deficient mice. Cell. 91:197-208.

11. Sugimoto, Y., et al. Failure of parturition in mice lacking the prostaglandin F receptor. Science. 277:681-683.

12. Murata, T., et al. 1997. Altered pain perception and inflammatory response in mice lacking prostacyclin receptor. Nature. 388:678-682.

13. Nguyen, M., et al. 1997. The prostaglandin receptor $\mathrm{EP}_{4}$ triggers remodelling of the cardiovascular system at birth. Nature. 390:78-81.

14. Hirata, M., et al. 1991. Cloning and expression of cDNA for a human thromboxane $\mathrm{A}_{2}$ receptor. Nature. 349:617-620.

15. Namba, T., et al. 1992. Mouse thromboxane $A_{2}$ receptor: cDNA cloning, expression and Northern blot analysis. Biochem. Biophys. Res. Commun. 184:1197-1203

16. Fleming, E.F., et al. 1998. Urinary concentrating function in mice lacking EP3 receptors for prostaglandin $\mathrm{E}_{2}$. Am. J. Physiol. 275:F955-F961.

17. Lee, J.B., and Attallah, A.A. 1975. Renal prostaglandins. Nephron. 15:350-368.

18. Mohn, A., and Koller, B.H. 1995. Genetic manipulation of embryonic stem cells. In DNA cloning 4. D.M. Glover and B.D. Hames, editors. Oxford University Press. New York, NY. 143-184.

19. Krege, J.H., Hodgin, J.B., Hagaman, J.R., and Smithies, O. 1995. A noninvasive computerized tail-cuff system for measuring blood pressure in mice. Hypertension. 25:1111-1115.

20. Oliver, M.I., Best, C.F.K.H.S., Arendshorst, W.J., Smithies, O., and Coffman, T.M. 1997. Angiotensin II responses in AT1A receptor-deficient mice: a role for AT1B receptors in blood pressure regulation. Am. J. Physiol. 272:F515-F520.

21. Thomas, D.W., et al. 1998. Coagulation defects and altered hemodynamic responses in mice lacking receptors for thromboxane $\mathrm{A}_{2}$. J. Clin. Invest. 102:1194-2001.

22. Tsafriri, A. 1995. Ovulation as a tissue remodelling process. In Tissue renin-angiotensin systems. A.K. Mukhipadhyay and M.K. Raizada, editors. Plenum Press. New York, NY. 121-140.

23. Schuetz, A.W., and Dubin, N.H. 1981. Progesterone and prostaglandin secretion by ovulated rat cumulus cell-oocyte complexes. Endocrinology. 108:457-463.

24. Mandl, J.P. 1972. The effect of prostaglandin $\mathrm{E}_{1}$ on rabbit sperm transport in vivo. J. Reprod. Fertil. 31:263-269.

25. Poulos, A., Voglmayr, J.K., and White, I.G. 1973. Phospholipid changes in spermatozoa during passage through the genital tract of the bull. Biochim. Biophys. Acta. 306:194-202.

26. Hayashi, S., Noda, Y., Matsumoto, H., and Mori, T. 1987. Fertilization of unovulated mature eggs following indomethacin administration in mice. Gamete Res. 18:291

27. Al-Bazzaz, F., Yadava, V.P., and Westenfelder, C. 1981. Modification of $\mathrm{Na}$ and $\mathrm{Cl}$ transport in canine tracheal mucosa by prostaglandins. Am. J. Physiol. 240:F101-F105.

28. Breyer, M.D., et al. 1998. Regulation of renal function by prostaglandin E receptors. Kidney Int. 54(Suppl. 67):S88-S94.

29. Nemoto, K., Pilbeam, C.C., Bilak, S.R., and Raisz, L.G. 1997. Molecular cloning and expression of the rat prostaglandin $\mathrm{E}_{2}$ receptor of the $\mathrm{EP}_{2}$ subtype. Prostaglandins. 54:713-725.

30. Katsuyama, M., et al. 1995. The mouse prostaglandin E receptor $\mathrm{EP}_{2}$ subtype: cloning expression and Northern blot analysis. FEBS Lett. 372:151-156.

31. Lee, J.B., Covino, B.G., Takman, B.H, and Smith, E.R. 1965. Renomedullary vasodepressor substance, medullin: isolation, chemical characterization, and physiological properties. Circ. Res. 17:57-77.

32. Daniels, E.G., Hinman, J.W., Leach, B.E., and Muirhead, E.E. 1967. Identification of prostaglandin $\mathrm{E}_{2}$ as the principal vasodepressor lipid of rabbit renal medulla. Nature. 215:1298-1299.

33. Hockel, G.M., and Cowley, A.W. 1980. Role of the renin-angiotensin system in prostaglandin E2-induced hypertension. Hypertension. 2:529-537.

34. Imanishi, M., et al. 1989. Aspirin lowers blood pressure in patients with renovascular hypertension. Hypertension. 14:461-468.

35. Hackenthal, E., Paul, M., Ganten, D., and Taugner, R. 1990. Morphology, physiology, and molecular biology of renin secretion. Physiol. Rev. 70:1067-1116.

36. Jensen, B.L., Schmid, C., and Kurtz, A. 1996. Prostaglandins stimulate renin secretion and renin mRNA in mouse renal juxtaglomerular cells. Am. J. Physiol. 271:F659-F669.

37. Tanimoto, K., et al. 1994. Angiotensinogen-deficient mice with hypotension. J. Biol. Chem. 269:31334-31337. 